\author{
Cadernos de \\ ESTUDOS LINGǗ̈ST|COS - (56.1), Campinas, Jan./Jun. 2014
}

\title{
ASSIMETRIAS NA HARMONIA VOCÁLICA EM PORTUGUÊS DO BRASIL
}

\author{
FILOMENA SANDALO* \\ UNICAMP, CNPq. \\ MARIA BERNADETE ABAURRE** \\ UNICAMP, CNPq.
}

\section{INTRODUÇÃO}

É comum, em processos de harmonia vocálica, que a vogal /a/ não se comporte como um gatilho. Este é o caso, por exemplo, do iorubá (cf. Archangeli e Pulleyblank 1989). Embora o iorubá apresente harmonia vocálica envolvendo vogais baixas, $\mathrm{o} / \mathrm{a} /$ não participa do processo. Fato similar ao do iorubá ocorre em alguns dialetos do português do Brasil (PB). Abaurre e Sandalo (2009) observam que a vogal /a/ não provoca harmonia de baixa nas pretônicas em variedades de Belo Horizonte, Vitória e Goiânia, embora as vogais médias baixas sejam gatilho para a harmonia. Os dados trabalhados por Kentowicz e Sandalo (2011), no entanto, permitem identificar ocorrências dessa harmonia na variedade de Recife, onde as pretônicas /e, o/ podem sofrer abaixamento antes de /a/ tônico (cf. 'pelado', 'colada', 'selada'). Sandalo et al. (2013) observam que a vogal /a/ é gatilho de harmonia de pretônicas também em Porto Alegre.

Neste trabalho, buscamos entender o comportamento da vogal /a/ na tônica como gatilho ou não de harmonia em PB. À luz da Teoria da Dispersão (Flemming 2004), nossa hipótese é de que o comportamento fonológico do /a/ em relação a sua participação em harmonia é dependente da dispersão acústica desta vogal com relação ao sistema vocálico do qual faz parte. Assumimos, com Flemming, que um espaço acústico mais aglutinado minimiza a percepção dos contrastes e, com Walker (2005), que a harmonia de altura é uma estratégia de realce para maximizar contrastes de altura. Assim, propomos que a função da harmonia em Recife e Porto Alegre é realçar a qualidade baixa do /a/, uma vez que os contrastes de altura foram minimizados na diminuição do espaço vocálico nesses sistemas. Ou seja, quando há uma maior distância fonética entre

\footnotetext{
* sandalo@iel.unicamp.br

** bernadete.abaurre@gmail.com
} 
espaços de contraste (i.e. o /a/ se encontra bastante mais baixo, na ponta do triângulo acústico em relação às outras vogais), não há harmonia com /a/. Por outro lado, quando a distância fonética entre espaços de contraste é menor (i.e. o /a/ se encontra mais próximo das outras vogais baixas, com os espaços acústicos dessas vogais mais aglutinados), há harmonia com /a/.

Este texto está organizado da seguinte maneira. Em 2, apresentamos nossa metodologia para verificar acusticamente a ocorrência de harmonia de vogais pretônicas com tônicas. Em 3, apresentamos os resultados de estudos sobre harmonia e mencionamos a assimetria relacionada à vogal /a/ em dados de São Paulo, Belo Horizonte e Recife. Em 4, elaboramos nossa hipótese explicativa e, em 5, verificamos o poder de previsão de nossa hipótese com base em dados de Porto Alegre.

\section{METODOLOGIA}

Este é um estudo experimental que consistiu em elicitar palavras paroxítonas trissilábicas com as possíveis combinações de vogais do PB na pretônica e na tônica (5 vogais na pretônica e sete na tônica; 35 combinações no interior das palavras). Cada combinação foi representada no corpus por um grupo de quatro ou cinco palavras (170 palavras) que foram lidas em uma frase veículo: 'Ela disse _ devagar'. Os dados foram obtidos em dois momentos. Em primeiro lugar, utilizaram-se os dados de Kenstowicz e Sandalo (2011) para a análise de fala de São Paulo, Belo Horizonte e Recife. O mesmo experimento foi posteriormente replicado por Sandalo et al. (2013) para a análise da fala de Salvador e Porto Alegre. Usaremos aqui apenas os dados referentes a Porto Alegre, já que Salvador não apresenta um dialeto harmonizante com vogais baixas na tônica, segundo esse trabalho.

Os dados que utilizaremos do primeiro experimento são de quatro falantes (PB1, PB2, PB3 e PB4) de regiões distintas do Brasil: Recife (1 informante do sexo masculino), Belo Horizonte ( 2 informantes, um do sexo masculino e um falante do sexo feminino) e Campinas (1 falante do sexo feminino), e os dados do segundo experimento são de um falante do sexo masculino de Porto Alegre (PB5).

Para atestar a ocorrência (ou não ocorrência) de harmonia em um dado conjunto de dados produzidos em contexto experimental, observou-se a correlação entre F1 (o correlato acústico de altura vocálica) da vogal da sílaba tônica e F1 da pretônica. Havendo correlação, afirma-se que há harmonia.

Para exemplificar a ocorrência de um dado de harmonia, vejamos a relação entre as pretônicas e tônicas a partir dos valores médios de F1 das vogais pretônicas de PB4:

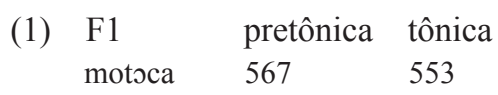


Observe a sequência de vogais [o-o] na palavra 'motoca' em (1), acima. O valor médio de F1 para uma vogal pretônica média posterior para este sujeito é de $450 \mathrm{~Hz}$. No entanto, nesta palavra, que tem uma vogal baixa na tônica, o valor de F1 da vogal pretônica é de $567 \mathrm{~Hz}$ (isto é, de uma vogal posterior baixa), aproximando-se ao valor de $553 \mathrm{~Hz}$ da tônica. Na Figura 1 , apresentamos os valores médios de F1 das vogais tônicas segundo o estudo de Escudero e al. (2009), nos quais nos baseamos para observar que a realização da pretônica em (1), com valor de $567 \mathrm{~Hz}$, indica ocorrência de uma vogal média baixa em correlação com a tônica, o que evidencia, portanto, harmonia.

\begin{tabular}{|c|c|c|c|c|c|c|c|c|}
\hline & & /i/ & /e/ & $/ \mathcal{E} /$ & $/ \mathrm{a} /$ & $10 /$ & $/ 0 /$ & $/ \mathrm{u} /$ \\
\hline \multirow[t]{4}{*}{$\mathrm{F} 1(\mathrm{~Hz})$} & $\mathrm{F}$ & 307 & 425 & 646 & 910 & 681 & 442 & 337 \\
\hline & & (1.198) & (1.082) & (1.076) & (1.078) & (1.087) & (1.094) & (1.192) \\
\hline & M & 285 & 357 & 518 & 683 & 532 & 372 & 310 \\
\hline & & $(1.077)$ & (1.077) & (1.089) & $(1.095)$ & (1.060) & (1.100) & (1.070) \\
\hline
\end{tabular}

Figura 1: Valores médios de F1 nas tônicas do PB (Escudero e al. 2009)

\section{RESULTADOS DO ESTUDO DE HARMONIA COM /A/ EM DADOS DE SÃO PAULO, BELO HORIZONTE E RECIFE}

Os dados de PB1, PB2, PB3 e PB4 evidenciam harmonia entre vogais pretônicas médias altas e vogais tônicas médias baixas, que se manifesta de diferentes maneiras nos dialetos. Apenas PB4 e PB5, porém, apresentam também harmonia com /a/. Nesta seção vamos discutir os dados dos primeiros quatro dialetos. Na seção 5, voltaremos a considerar os dados de PB5.

Os valores médios de F1 das pretônicas dos sujeitos de Kenstowicz e Sandalo (2011) (PB1, PB2, PB3, PB4) estão na Figura 2.

\begin{tabular}{cccccc}
\hline & i & u & e & o & a \\
\hline BP1 & 343 & 387 & 543 & 528 & 899 \\
BP2 & 419 & 456 & 542 & 551 & 812 \\
BP3 & 284 & 360 & 413 & 457 & 787 \\
BP4 & 227 & 386 & 478 & 450 & 595
\end{tabular}

Figura 2: Valores médios de F1 das vogais pretônicas de PB1, PB2, PB3 e PB4

(Kenstowicz e Sandalo 2011)

Abaixo (Fig. 3), apresentamos os valores de F1 de cada combinação de vogais médias na pretônica com todas as vogais do português na tônica em BP2, para exemplificar um dialeto que não harmoniza com /a/. Conforme mostrado acima, o valor médio de uma pretônica média alta para PB2 é de $551 \mathrm{~Hz}$ para [o] e $542 \mathrm{~Hz}$ para [e]. Em negrito, na Figura 3, mostramos que o valor de F1 da vogal baixou significativamente diante de uma vogal média baixa (embora para PB2 não tenha 
havido abaixamento quando a sequência foi e- 0 , diferentemente do que ocorreu nos dados de outros falantes), mas nunca houve abaixamento diante de /a/.

\begin{tabular}{|l|l|}
\multicolumn{1}{l|}{ PB2 } & \multicolumn{1}{l|}{ F1 da pretônica } \\
\hline o-á & 540 \\
\hline o-ó & 551 \\
\hline o-é & 520 \\
\hline o-ó & 659 \\
\hline e-ć & 664 \\
\hline
\end{tabular}

\begin{tabular}{|l|l|}
\hline e-á & 572 \\
\hline e-ó & 529 \\
\hline e-é & 503 \\
\hline e-ó & 543 \\
\hline e-ć & 684 \\
\hline
\end{tabular}

Figura 3: Valores de F1 da pretônica em PB2 considerando-se diferentes sequências de vogais (Kenstowicz e Sandalo 2011)

O fenômeno de harmonia com /a/, no entanto, foi atestado para os dados de PB4, segundo a metodologia descrita, como se pode verificar na Figura 4.

\begin{tabular}{|c|c|c|c|c|c|}
\hline \multicolumn{2}{|c|}{ F1 pretônica } & \multicolumn{2}{|c|}{ F1tônica } & \multirow{2}{*}{$\begin{array}{c}\text { F1pretônica } \\
323\end{array}$} & \multirow{2}{*}{$\begin{array}{c}\text { F1tônica } \\
315\end{array}$} \\
\hline pepino & 293 & 386 & popula & & \\
\hline fedida & 300 & 300 & modula & 330 & 470 \\
\hline ferida & 312 & 310 & fodida & 336 & 300 \\
\hline tereza & 384 & 396 & sossega & 357 & 558 \\
\hline tesuda & 401 & 399 & cozida & 367 & 318 \\
\hline ferido & 402 & 315 & bonita & 387 & 342 \\
\hline perigo & 405 & 337 & polida & 412 & 308 \\
\hline cereja & 406 & 423 & moleque & 425 & 565 \\
\hline selada & 521 & 630 & toledo & 495 & 424 \\
\hline pelado & 525 & 651 & boboca & 506 & 586 \\
\hline vencro & 527 & 566 & folhada & 508 & 617 \\
\hline medonha & 545 & 674 & topete & 516 & 525 \\
\hline rebote & 558 & 549 & lotada & 516 & 636 \\
\hline pelote & 560 & 569 & colada & 524 & 643 \\
\hline leleca & 561 & 533 & cocada & 527 & 633 \\
\hline decote & 566 & 573 & mocada & 534 & 630 \\
\hline meleca & 566 & 549 & colcta & 534 & 567 \\
\hline
\end{tabular}




$\begin{array}{llllll}\text { metade } & 567 & 614 & \text { lorota } & 536 & 578 \\ \text { melado } & 582 & 614 & \text { rodela } & 551 & 525 \\ \text { retoque } & 584 & 566 & \text { motoca } & 567 & 553 \\ & & & \text { mococa } & 599 & 615\end{array}$

Figura 4: Alguns valores de F1 na tônica e pretônica de PB4 (Kenstowicz e Sandalo 2011)

Observe as palavras 'rodela', 'motoca' e 'melado', exemplares de ocorrência de harmonia com as duas médias baixas /E/ e / / / e com a baixa /a/ tônicas, segundo a metodologia aqui utilizada. Os valores de F1 para a pretônica nos três itens é $551 \mathrm{~Hz}, 567 \mathrm{~Hz}$ e $582 \mathrm{~Hz}$ respectivamente. Vale ressaltar que o valor médio de $\mathrm{F} 1$ das vogais pretônicas médias fechadas de PB4 (cf. Figura 2) é de $478 \mathrm{~Hz}$ para o [e] e de $478 \mathrm{~Hz}$ para o [o]. Os valores da pretônica de 'rodela' e 'motoca' para BP4 foram abaixados, portanto, conforme se pode atestar na Figura 4. Em 'melado', onde a tônica é /a/, também se pode afirmar que houve harmonia, pois o valor de F1 da vogal pretônica é de $582 \mathrm{~Hz}$, superando o valor médio de um [e] pretônico, que é de $450 \mathrm{~Hz}$ para BP4. Logo, os dados não deixam dúvidas de que, segundo a metodologia utilizada, há harmonia com /a/ nos dados analisados de Recife. Notese que nos dados de PB4 nunca houve abaixamento de vogal pretônica antes de uma vogal fechada, como pode ser observado na Figura 4.

\section{HIPÓTESE EXPLICATIVA PARA A HARMONIA E NÃO HARMONIA COM /A/ BASEADA NA TEORIA DA DISPERSÃO}

A Teoria da Dispersão (Flemming 2004) é inspirada na Teoria da Dispersão Adaptativa (Lindblom 1986). Segundo esta perspectiva, a seleção de contrastes fonológicos é orientada por 3 metas funcionais:

1. Maximizar a distintividade dos contrastes em um dado espaço acústico

2. Minimizar o esforço articulatório

3. Maximizar o número de contrastes

Essas metas, entretanto, podem conflitar, conforme esquematizado abaixo. Quanto maior for a distância entre as categorias distribuídas em um espaço, maior a distintividade. Quanto maior a aproximação entre as categorias, menor o esforço articulatório. A Figura 5 ilustra o conflito com três distribuições diferentes de categorias em um mesmo espaço.

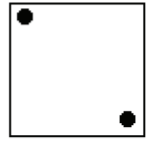

(a)

Two categories Most separation Less separation More effort

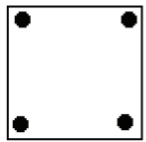

(b) More effort

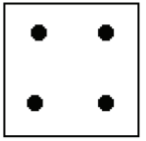

(c)

Four categories

Least separation

Less effort

Figura 5: Espaço, categorias e conflitos (Flemming 2004) 
Nas palavras do autor, "(...)if we assume that sounds in the periphery of the space involve greater effort than those in the interior, than, to avoid effortful sounds it is necessary to restrict sounds to a reduced area of the space, thus the contrasts will be less distinct." (Flemming 2004: 237)

Nossa explicação: o comportamento fonológico do /a/ em relação a sua participação em harmonia é dependente da dispersão acústica desta vogal com relação ao sistema vocálico do qual faz parte. Abaixo, apresentamos os gráficos com as dispersões acústicas das vogais de cada informante (PB1, PB2, PB3 e PB4).

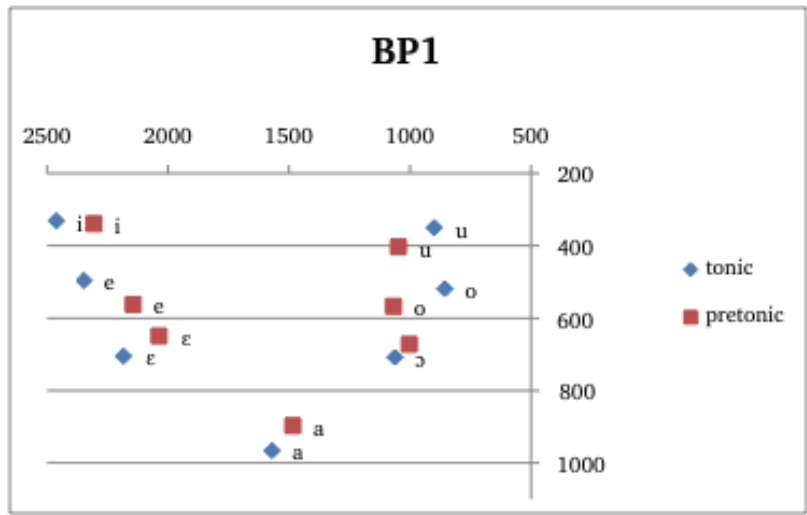

Figura 6: Espaço acústico das vogais de PB1, São Paulo (Kenstowicz e Sandalo 2011)

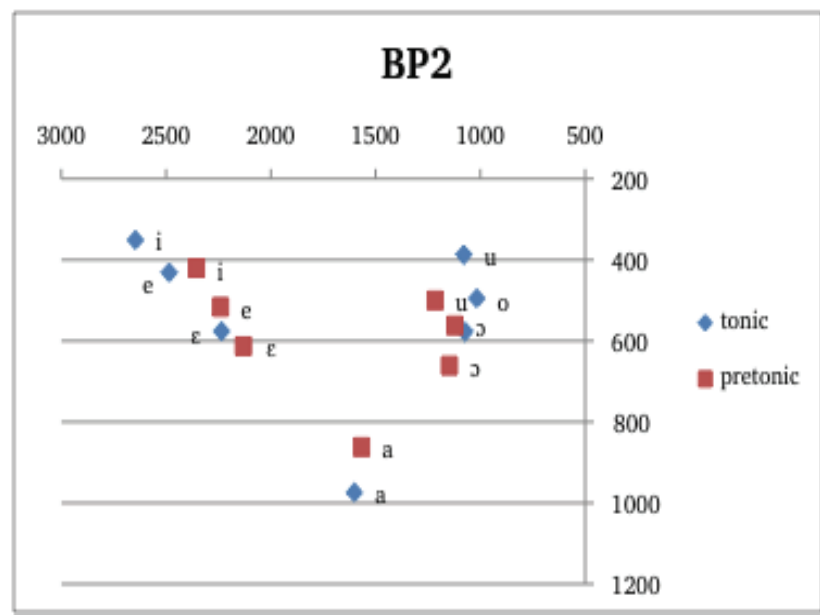

Figura 7: Espaço acústico das vogais de PB2, Belo Horizonte (Kenstowicz e Sandalo 2011) 


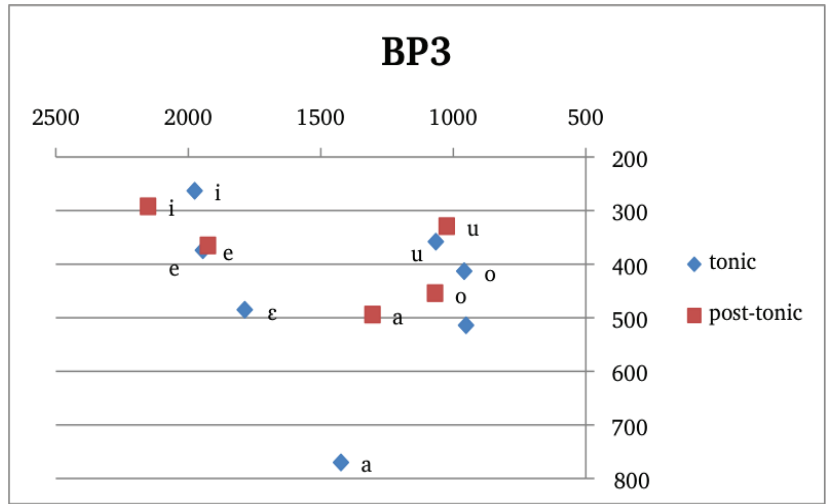

Figura 8: Espaço acústico das vogais de PB3, Belo Horizonte

(Kenstowicz e Sandalo 2011)

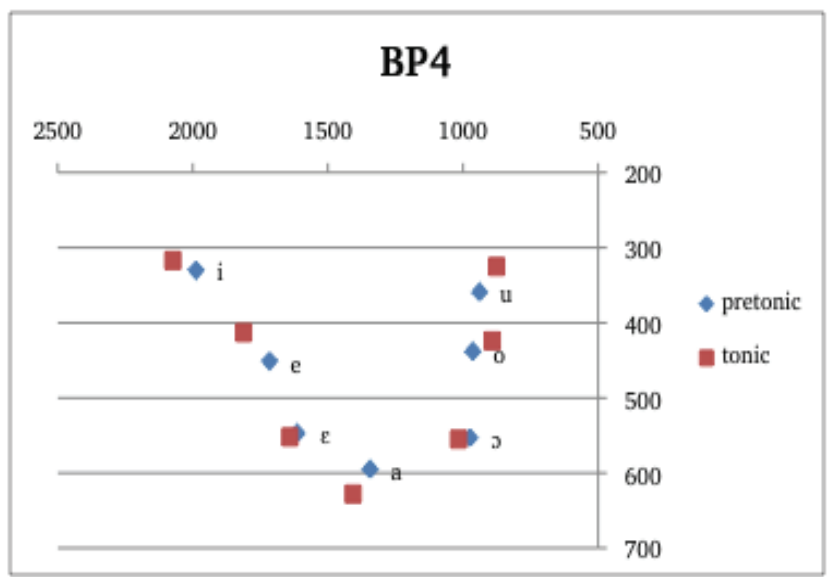

Figura 9: Espaço acústico das vogais de PB4, Recife (Kenstowicz e Sandalo 2011)

Observe nas Figuras 7-9 que Recife apresenta uma configuração do espaço vocálico diferente dos demais sistemas, no que diz respeito à distância entre a vogal baixa /a/ e as médias baixas. Recife apresenta um sistema mais polarizado para as baixas. Configuração similar para Recife foi também observada para as vogais pretônicas no estudo de Callou et al. (2013:84), em que apresentam os espaços acústicos das vogais do PB para as cinco capitais do projeto NURC (Recife, São Paulo, Salvador, Rio de Janeiro e Porto Alegre).

Se assumirmos, com Walker (2005), que a harmonia de altura é uma estratégia de realce para maximizar contrastes de altura, podemos propor que a função da harmonia em PB4 (Recife) é de realçar a qualidade baixa do /a/, uma vez que os contrastes de altura foram minimizados na diminuição do espaço vocálico neste sistema (cf. Figura 9). 
Mais especificamente, nossa hipótese é de que:

- Maior distância fonética entre espaços de contraste (i.e. o /a/ se encontra bastante mais baixo, na ponta do triângulo acústico em relação às outras vogais): não há harmonia com $/ \mathrm{a} /$.

- Menor distância fonética entre espaços de contraste (i.e. o /a/ se encontra mais aglutinado às alturas das outras vogais baixas, ou seja, os espaços acústicos das vogais baixas são mais aglutinados): há harmonia com /a/.

Na próxima seção, procuraremos testar nossa hipótese verificando dados de outra variedade do PB, PB5.

\section{PORTO ALEGRE}

Decidimos testar dados de Porto Alegre porque, segundo Callou et al. (2013), este dialeto apresenta a distribuição de vogais no espaço acústico semelhante ao que se observa em Recife "[...] o sistema menos polarizado é o de Porto Alegre, acompanhado de perto por Recife, em que as vogais altas e a baixa estão mais próximas." (Callou et al. 2013:84). Baseadas nesta observação, obtivemos os valores e a configuração das vogais de Porto Alegre, que apresenta o espaço acústico da Figura 10, em que as vogais em preto são as tônicas e as vogais em vermelho são as pretônicas:

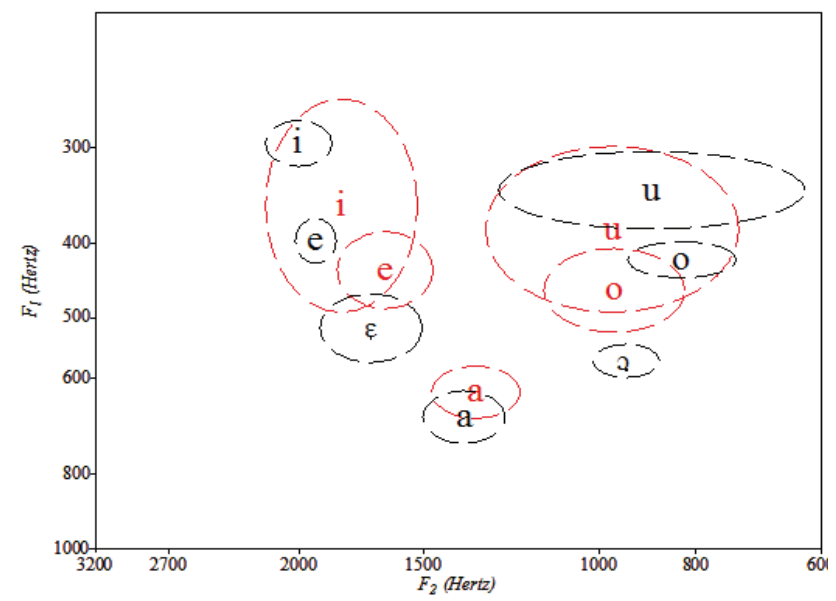

Figura 10: Espaço acústico das vogais do falante de Porto Alegre (Sandalo et al. 2013)

Como pode ser observado na Figura 10, de fato, as vogais médias baixas e a vogal baixa estão mais aproximadas no espaço acústico do que se observa em Belo Horizonte e São Paulo, apresentando, portanto, uma configuração semelhante à de Recife em relação a estas vogais (cf. Figuras 7-9). 
Abaixo, apresentamos os valores médios de F1 das vogais tônicas e pretônicas de PB5 (Sandalo et al. 2013) para aplicação de nossa metodologia para verificação da ocorrência de harmonia vocálica. Vamos agora verificar se este dialeto apresenta harmonia com baixas incluindo a vogal /a/, para testar nossa hipótese de que a configuração do espaço acústico determina a ocorrência ou não de harmonia com /a/.

\begin{tabular}{c|ccccccc}
\hline & $\mathrm{i}$ & $\mathrm{e}$ & $\mathrm{E}$ & $\mathrm{a}$ & $\mathrm{O}$ & $\mathrm{o}$ & $\mathrm{u}$ \\
\hline POA & 296 & 397 & 518 & 675 & 570 & 420 & 343 \\
\hline
\end{tabular}

Figura 11: Valores médios de F1 das vogais tônicas (PB5) do sujeito de Sandalo et al. (2013)

\begin{tabular}{c|ccccc}
\hline & i & e & a & o & u \\
\hline POA & 387 & 436 & 627 & 463 & 400 \\
\hline
\end{tabular}

Figura 12: Valores médios de F1 das vogais pretônicas (PB5) do sujeito de Sandalo et al. (2013)

Um recorte dos dados de PB5 segue abaixo. O fenômeno foi atestado segundo a metodologia descrita para os dados de PB5, como se pode verificar na Figura 13:

\begin{tabular}{cccccc}
\hline Palavra & F1- Pretônica & F1 - Tônica & Palavra & F1- Pretônica & F1 - Tônica \\
\hline sossega & 284 & 512 & deboche & 466 & 453 \\
fofoca & 497 & 483 & teteca & 332 & 483 \\
moçada & 362 & 656 & melado & 585 & 691 \\
lorota & 474 & 480 & pelote & 531 & 465 \\
motoca & 521 & 458 & leleca & 512 & 493 \\
folhada & 409 & 669 & pedaço & 519 & 675 \\
rodela & 516 & 424 & meleca & 470 & 561 \\
topete & 503 & 479 & metade & 500 & 674 \\
coleta & 530 & 482 & peteca & 478 & 479 \\
boboca & 487 & 471 & rebote & 502 & 477 \\
mococa & 509 & 474 & selada & 517 & 703 \\
lotada & 502 & 678 & venero & 374 & 454 \\
colado & 524 & 675 & decote & 482 & 482 \\
& & & retoque & 487 & 472 \\
\hline
\end{tabular}

Figura 13: Dados de Porto Alegre (Sandalo et al. 2013)

Observe as palavras 'rodela', 'motoca' e 'melado', exemplares de ocorrência de harmonia com as duas médias baixas / $\mathcal{E} /$ e / $/$ e com a baixa /a/, segundo a metodologia aqui utilizada. Os valores de F1 para a pretônica nos três itens é $516 \mathrm{~Hz}, 521 \mathrm{~Hz}$ e $585 \mathrm{~Hz}$, respectivamente. Vale ressaltar que o valor médio de 
F1 das tônicas é de $518 \mathrm{~Hz}$ para o /E/ e de $570 \mathrm{~Hz}$ para o / $/$, para o falante de Porto Alegre (cf. Sandalo et al. 2013), na Figura 11. Os valores da pretônica de 'rodela' e 'motoca', portanto, são de [o] e não de [o], cujo F1 apresenta valor médio de $420 \mathrm{~Hz}$. A diferença entre os valores da vogal média baixa e da média alta é próxima a $100 \mathrm{~Hz}$ para os dois itens. Em 'melado', também pode-se afirmar que houve harmonia, pois o valor de F1 da vogal pretônica é de $585 \mathrm{~Hz}$, superando o valor médio de um [e] pretônico em $188 \mathrm{~Hz}$. Logo, os dados não deixam dúvidas de que há harmonia, também nos dados analisados de Porto Alegre, com as três vogais baixas, embora o fenômeno seja variável, isto é, nem sempre a harmonia ocorre, como nos outros dialetos considerados.

A harmonia com /a/ foi, portanto, atestada acusticamente em Porto Alegre: há abaixamento da vogal média diante de /a/ quando observados os valores de F1 da tônica e da pretônica.

\section{CONCLUSÃO}

Os dados analisados de Porto Alegre corroboram, portanto, a nossa hipótese, retomada abaixo:

- Maior distância fonética entre espaços de contraste (i.e. o /a/ se encontra bastante mais baixo, na ponta do triângulo acústico em relação às outras vogais): não há harmonia com /a/.

- Menor distância fonética entre espaços de contraste (i.e. o /a/ se encontra mais próximo das outras vogais baixas, ou seja, os espaços acústicos das vogais baixas são mais aglutinados): há harmonia com /a/.

Obviamente, outros estudos, baseados em dados de outras variedades de PB, e incluindo as necessárias análises de significância estatística que não foram aqui realizadas, poderão permitir conclusões mais robustas que atestem que a maior ou menor proximidade entre as baixas no interior do espaço acústico pode, de fato, prever o comportamento assimétrico da vogal /a/ no processo de harmonia vocálica.

\section{REFERÊNCIAS BIBLIOGRÁFICAS}

ARCHANGELI, Diana e Douglas Pulleyblank. 1989. Yoruba Vowel Harmony. Linguistic Inquiry, 20.2: $173-217$.

ABAURRE, Maria Bernadete e Filomena Sandalo. 2009. Representação e subespecificação de vogais no português. Cadernos de Pesquisas em Linguística, 4.1: 21-40. Porto Alegre.

CALLOU, Dinah; João Antonio de Moraes e Yonne Leite. 2013. As vogais orais: um estudo acústicovariacionista, em: M.B. Abaurre (org.), Gramática do português culto falado no Brasil, vol VII: A construção fonológica da palavra. São Paulo, Editora Contexto: 75-93. 
ESCUDERO, Paola, Paul Boersma, Andreia Rauber e Ricardo Bion. 2009. A cross-dialect acoustic description of vowels: Brazilian and European Portuguese. Journal of the Acoustical Society of America, 126: 1379-1393.

FLEMMING, Edward. 2004. Contrast and perceptual distinctiveness, em: B. Hayes; R. Kirchner e D. Steriade (orgs), Phonetically Based Phonology. Cambridge, Cambridge University Press: 232-76.

KENSTOWICZ, Michael e Filomena Sandalo. 2011. Vowel Harmony and Dispersion in Brazilian Portuguese. Apresentação no MIT Phonology Circle, 8 de março de 2011.

LINDBLOM, Björn. 1986. Phonetic universals in vowel systems, em J. Ohala e J. Jaeger, Experimental phonology, Orlando, Academic Press: 13-44.

SANDALO, Filomena; Maria Bernadete Abaurre e Magnun Rochel Madruga. 2013. Dispersão e harmonia vocálica em dialetos do português do Brasil. Organon, 28.54: 13-30. Universidade Federal do Rio Grande do Sul.

WALKER, Rachel. 2005. Weak triggers in vowel harmony. Natural Language and Linguistic Theory, 23:917-989. 